\title{
¿Cómo conforma el profesorado universitario su alta resiliencia? Un estudio de casos
}

\author{
How do University Professors build High Resilience? Case Studies
}

\section{Como se conforma em professores universitarios sua alta resiliência? Um estudo de casos}

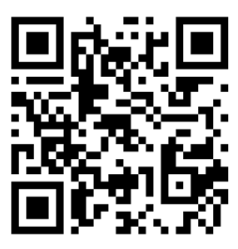

Teresa de Jesús Sierra-Molina

Universidad Marista de Mérida

Yucatán, México

tsierra@marista.edu.mx

https://orcid.org/0000-0002-6915-9824

Dora Esperanza Sevilla-Santo

Universidad Autónoma de Yucatán

Facultad de Educación

Yucatán, México

dora.sevilla@correo.uady.mx

https://orcid.org/0000-0001-9781-6783

Recibido • Received • Recebido: 25 / 10 / 2019

Corregido • Revised • Revisado: 21 / 06 / 2021

Aceptado • Accepted • Aprovado: 25 / 08 / 2021

\begin{abstract}
Resumen
Introducción. La resiliencia es entendida desde una perspectiva triádica, como la creación de nuevas estrategias a partir de la necesidad de resistir en una interacción de características personales, con un ambiente familiar estable, así como con un contexto que apoye. Objetivo. conocer cómo el profesorado universitario conforma su alta resiliencia, para que tanto este como las IES puedan disminuir factores de riesgo, promover mecanismos que permitan altos niveles de resiliencia y una mayor permanencia en la labor docente. Metodología. Enfoque cualitativo de alcance descriptivo, con ocho profesores y profesoras de ingeniería y psicología de IES públicas y privadas del sureste mexicano, que formaron parte de un trabajo doctoral con enfoque mixto Resultados. Se identifica la importancia de aspectos profesionales y de la labor docente en la conformación de la alta resiliencia, ya que actúan como factores protectores y de riesgo, tanto internos como externos, que permiten mantener la alta resiliencia. Le siguen, en importancia, aspectos personales que funcionan como protectores y de riesgo, como el compaginar la docencia con la maternidad de hijas e hijos pequeños. En tercer lugar, se identifican aspectos institucionales percibidos casi en igual medida como protectores y de riesgo. Por último, los aspectos sociales son más percibidos por el personal docente de ingeniería y por el de tiempo completo. Conclusiones. Se corrobora la dinámica y bidireccionalidad de estos aspectos en la conformación de alta resiliencia que, según las experiencias del personal docente, son percibidos como factores protectores que mitigan los muchos riesgos en forma de retos que la docencia actual les presenta.
\end{abstract}

Palabras claves: Resiliencia; educación superior; personal académico docente; reforma de la educación. 
http://doi.org/10.15359/ree.25-3.16

http://www.una.ac.cr/educare

educare@una.ac.cr

\begin{abstract}
Introduction. Resilience is understood under a triadic perspective as the creation of new strategies from the need to resist in the interaction of personal characteristics, with a stable family environment and a supportive context. Objective. To know how university teachers build their high resilience so that they and higher education institutions (HEI) can reduce risk factors and promote mechanisms allowing high levels of resilience and greater time working as teachers. Methodology. A qualitative approach of descriptive scope for eight professors of engineering and psychology of public and private HEI from the Mexican southeast who were part of a doctoral work project with a mixed approach. Results. First, the importance of professional aspects and the teaching work in building high resilience is identified since these two elements act as protective and risk factors, both internal and external, that allow maintaining resilience. Second, next in importance are personal aspects that function as protectors and risk factors, such as combining teaching with the motherhood of young children. Third, institutional aspects are identified, which are perceived, almost equally, as protective and risky. Finally, social aspects are more perceived by the engineering teaching personnel and the full-time working staff. Conclusions. The dynamics and bidirectionality of these aspects to build higher resilience levels are corroborated; according to the experiences of the teaching staff, these aspects are perceived as protective factors that mitigate many risks in the form of challenges that current teaching poses to teachers.
\end{abstract}

Keywords: Resilience; higher education; teaching profession; educational reform.

\begin{abstract}
Resumo
Introdução. Resiliência é entendida em uma perspectiva triádica, como a criação de novas estratégias a partir da necessidade de resistir em uma interação de características pessoais, com um ambiente familiar estável, bem como com um contexto de apoio. Objetivo. Saber como os professores universitários moldam a sua elevada resiliência, para que tanto eles como as IES possam reduzir os fatores de risco, promover mecanismos que permitam elevados níveis de resiliência e uma maior permanência no trabalho. Metodologia. Uma abordagem qualitativa de modelo descritivo para oito professores de engenharia e psicologia de IES públicas e privadas do sudeste mexicano que fizeram parte de um trabalho de doutorado com abordagem mista. Resultados. Identifica-se a importância dos aspectos profissionais e do trabalho docente na conformação da alta resiliência, uma vez que atuam como fatores protetores e de risco, internos e externos, que permitem manter a resiliência. Segue-se em importância os aspectos pessoais que funcionam como protetores e fatores de risco, como a conjugação do ensino com a maternidade dos filhos pequenos. Terceiro, são identificados aspectos institucionais que são percebidos quase igualmente como protetores e arriscados. Por fim, os aspectos sociais são mais percebidos pelo corpo docente da engenharia e pelos de tempo integral. Conclusões. É corroborada a dinâmica e a bidirecionalidade desses aspectos na formação de alta resiliência, os quais, segundo as experiências dos docentes, são percebidos como fatores de proteção que mitigam os diversos riscos em forma de desafios que o ensino atual lhes apresenta.
\end{abstract}

Palavras-chave: Resiliência; ensino superior; corpo docente acadêmico; reforma educacional.

\title{
Hacia una definición de resiliencia
}

La resiliencia, como fenómeno, ha sido estudiada desde hace varias décadas y en distintos campos del conocimiento. Desde la epidemiología tiene sus antecedentes en los trabajos clásicos 
http://doi.org/10.15359/ree.25-3.16

http://www.una.ac.cr/educare educare@una.ac.cr

de los años sesenta de Emmy Werner, en los que describió la resiliencia como la característica de personas que se reponen a un trauma.

Más adelante, otros estudios comienzan a definir características comunes de las personas que se sobreponen a los riesgos. Diversas investigaciones (Grotberg (1996), Henderson y Milstein, 2003; Melillo, 2004) han cuestionado conceptos como: vulnerabilidad, sucesos desestabilizadores y resistencia; igualmente se han preguntado: ¿estos aspectos los traen las personas de nacimiento?, ¿se aprenden?, ¿qué es lo que se puede hacer para desarrollarlos?, ¿se pueden aplicar al ámbito educativo?

Ejemplo de lo anterior son las fuentes de la resiliencia identificadas por Grotberg (1996) Yo soy, yo estoy, yo puedo y yo tengo; así como los pilares de la resiliencia definidos por Melillo (2004): autoestima, insight o perspicacia, independencia, capacidad para relacionarse, iniciativa, humor y creatividad, moralidad y pensamiento crítico.

Más recientemente, la resiliencia ha sido asociada y estudiada en situaciones de riesgo ecológico, en arquitectura, urbanismo, trabajo social y, desde luego, la educación; ya que se incluye en su definición, desde una perspectiva triádica: a) los recursos que el individuo identifica como propios, b) la percepción de un ambiente familiar estable y, c) contar con redes sociales externas que estimulan el enfrentamiento adaptativo, como mencionan Capanna et al. (2015). Igualmente, se le describe, en la actualidad, como un proceso en el que se ve a la persona en resiliencia (Quintero Velásquez, 2005).

Para propósitos de este trabajo, se considera la resiliencia desde esta perspectiva, como un proceso dinámico emocional, cognitivo o sociocultural (Henderson Grotberg, 2006) de una persona o de un grupo para reconocer, enfrentar, transformar constructivamente experiencias adversas (Simpson, 2008) y desarrollar competencia social, académica y vocacional que es variable, nunca absoluta ni lograda para siempre (Villalobos Pérez-Cortés, 2009), que es específico de cada contexto y culturalmente sesgada (Ungar, 2003). Más aún, si como mencionan WongParodi et al. (2015), la persona o la comunidad hace uso de sus recursos y sale fortalecida.

\section{¿Cuál es la importancia de la resiliencia en la educación?}

En el campo de la educación, el fenómeno de la resiliencia es de reciente estudio, lo que queda claro en los recuentos de la historia de su abordaje que algunos estudios (Beigi, 2016; Quintero Velásquez, 2005) hacen acerca de su surgimiento como concepto en diversas áreas del conocimiento. Por ejemplo, en Estados Unidos, se identifican como pioneros en este campo los estudios de Henderson y Milstein (2003) acerca del papel que la escuela y el personal docente pueden jugar en la construcción de ambientes resilientes y en la mitigación de los factores de riesgo en los que se encuentran tanto estudiantes como profesorado, desde educación básica a superior. 
http://doi.org/10.15359/ree.25-3.16

http://www.una.ac.cr/educare

educare@una.ac.cr

Actualmente, debido a fenómenos como la globalización y el avance de las tecnologías, ser profesor o profesora es mucho más complejo que en épocas anteriores; por lo que es probable que el maestro y la maestra perciban nuevos retos y adversidades en su labor como docentes desde el entorno personal, profesional, social e institucional. Requiere cada vez adoptar y adaptarse a los cambios del contexto y poder responder a las demandas del alumnado, la sociedad, sus pares y las instituciones; lo que es más, se espera que pueda salir adelante y cambiar sus anteriores patrones de actuación como docente y que, cada vez que sea necesario, los vuelva a repensar y modificar.

En el contexto de las instituciones de educación superior (IES), se pueden identificar varias formas de reaccionar a los retos que la educación actual exige. Vicente de Vera y Gabari Gambarte (2019) plantean que algunos y algunas docentes presentan desgaste emocional y bournout, debido al exceso de tareas, las presiones de tiempo, conflictos con compañeros y compañeras, la percepción de falta de autonomía y además continuar con las otras labores docentes, sociales, profesionales, laborales y familiares.

Aunado a esto, Olivares-Faundéz y Villalta (2015) identifican altos niveles de burnout en profesionales que, además se desempeñan como docentes por horas. Estos datos coinciden con lo que Brooks (2016) refiere, cuando menciona la importancia de considerar la identidad docente dado que puede afectar su resiliencia, su compromiso y ulteriormente, su efectividad. Respecto a esto, Monereo y Domínguez (2014) señalan la importancia de las teorías implícitas y explícitas que el profesorado tiene acerca de los procesos institucionales de acreditación y evaluación, ya que estas influyen en la forma en la que el personal docente percibe riesgos en el ambiente o en sí mismo.

Dadas las situaciones antes mencionadas, sería importante que docentes e instituciones identifiquen estrategias constructivistas centradas en la solución del problema, en lugar de "estrategias de afrontamiento pasivas centradas en la emoción" como mencionan Vicente de Vera y Gabari Gambarte (2019, p. 129). Estrategias de afrontamiento a los múltiples requisitos institucionales que abonen, como menciona Carlos Guzmán (2011), al trato y la formación pedagógica de profesionales que se unen a la docencia sin una preparación pedagógica previa. Así mismo, es importante contar con apoyo personal de personal directivo (jefaturas) Villalobos Vergara y Assaél Budnik (2018) y personal que ayude con labores administrativas asociadas a la docencia. Esto coincide con lo mencionado también por Arnup y Bowles (2016) quienes destacan la importancia de que la cultura escolar sea percibida como un apoyo, de ahí la importancia de contar con la mirada del personal docente en el contexto educativo actual.

\section{El profesorado universitario y la resiliencia}

En las instituciones de educación superior (IES) se tiene en cuenta que el personal docente universitario es, ante todo, profesionales que, con base en la experiencia en su área o campo de trabajo, se ven en la posibilidad de formar a sus futuros grupos de colegas; sin embargo,

4

Teresa de Jesús Sierra-Molina y Dora Esperanza Sevilla-Santo

Los artículos de la Revista Electrónica Educare del Centro de Investigación y Docencia en Educación de la Universidad Nacional, Costa Rica, se comparten bajo términos de la Licencia Creative Commons: Reconocimiento, № Comercial, Sin Obra Derivada 3.0 Costa Rica. Las autorizaciones adicionales a las aquí delimitadas se pueden obtener en el correo: educare@una.cr 
http://doi.org/10.15359/ree.25-3.16

hasta ahora, estos sujetos profesionales que se desempeñan como docentes de universidades recibieron formación de una manera muy distinta al actual enfoque y requisitos con los que se pretende que propicien el aprendizaje de sus estudiantes en las materias que imparten.

Muchas de estas personas docentes reproducen con sus estudiantes aquellas acciones que heredaron de sus propios maestros y maestras, sin cuestionar si las formas 'convencionales' de enseñar son las que el alumnado de educación superior (ES) de hoy en día requiere para un adecuado aprendizaje y desempeño en la profesión; dadas las diferencias en sus formas de aprender, intereses, hábitos, manejo de la información y de las tecnologías. Como menciona Mérida Serrano (2006), "en el modelo de profesorado universitario tradicional que conocemos y en la mayoría de los casos, el centro de nuestra actividad profesional lo ocupa la preocupación por realizar una adecuada labor de enseñanza de los contenidos de nuestra asignatura" (p. 4), por lo que propiciar en el aula situaciones de aprendizaje que promueven habilidades, actitudes y valores puede resultar complejo para quien no recibió formación de esta manera.

En este contexto, la función del personal docente y su identidad se tornan cada vez más complejas e inciertas, debido a las múltiples tareas que se le encargan. Price et al. (2012) coinciden, además, en que las tendencias internacionales a estandarizar los procedimientos didácticos tienden a desanimar al personal docente, quienes perciben un aumento en los requerimientos institucionales mientras el pago sigue siendo muy bajo, lo que les lleva a cuestionar su identidad docente; con efectos poco deseables tanto en su desarrollo personal, en su desempeño como profesional de la educación, así como en la percepción de los riesgos que pueden estar afectando su resiliencia.

Sin embargo, la mayoría del profesorado que ha tomado los retos como una manera de mejorar en su práctica presenta menos resistencia, aprovecha sus recursos, pone en juego su creatividad y se adapta mejor a los cambios solicitados. Son maestras y maestros que afrontan activamente e identifican sus necesidades y valoran los apoyos que se les pueda brindar, se inscriben a los cursos de formación continua cuando sus instituciones los ofrecen y han intentado planear, enseñar y evaluar cómo se les ha solicitado, incorporando las innovaciones requeridas (Hargreaves, 1994).

Los anteriores son profesores y profesoras que, además, se apoyan en sus relaciones de trabajo y amistad con otro profesorado; sobre todo en momentos de altas demandas y ante situaciones dilemáticas, las cuales logran en conjunto resignificar la labor docente tal lo refieren Madariaga et al. (2014) y Vicente de Vera y Gabari Gambarte (2019). Maestras y maestros que al parecer las autoras del presente artículo demuestran características similares a las que conforman las fuentes de la resiliencia, lo que les permitiría identificar en qué medida junto a las otras personas en su entorno social e institucional tienen, son o pueden demostrar y desarrollar los pilares de la resiliencia como autoestima, independencia, iniciativa, humor, capacidad para relacionarse, creatividad, moralidad y pensamiento crítico; rasgos que los llevan a sobreponerse a las adversidades que la docencia actual presenta. 
http://doi.org/10.15359/ree.25-3.16

http://www.una.ac.cr/educare

educare@una.ac.cr

Los rasgos anteriores son percibidos como apoyos o factores protectores, y ponen en evidencia aspectos como la fortaleza y confianza que se tiene en la propia persona y qué tan competentemente se percibe socialmente, así como el apoyo de su familia y del personal docente de tiempo completo; factores significativos en la conformación de la resiliencia del profesorado universitario como se menciona en Sierra Molina et al. (2019).

Dichos factores protectores les permitirían hacer frente a las exigencias educativas, lo que se podría relacionar con niveles más altos de respuestas resilientes; situación que a su vez les podría diferenciar de los maestros y maestras que continúan trabajando sin incorporar los cambios y de aquellos grupos que abandonan la docencia (Hargreaves, 1994), tanto desde la perspectiva de la institución como la del alumnado con quienes conviven y a quienes forman cada semestre.

Estas situaciones las refiere Hong (2012, en Olivares-Faundéz y Villalta, 2015), al afirmar que "la resiliencia es un constructo explicativo en muchos casos de la permanencia de profesores en el sistema educativo" (p. 312); ya que, como mencionan Benítez-Corona y Barrón-Tirado (2018), han aprendido a observar las situaciones problemáticas (los riesgos), han articulado sus experiencias de vida y las han enfrentado con creatividad, capacidad de introspección, moralidad y pensamiento crítico que les ha permitido "emitir propuestas valiosas para la educación" (p. 16).

Cabe señalar que los mismos aspectos que para algunas personas docentes pueden fungir como factores protectores, para otras personas docentes podrían representar factores de riesgo a su resiliencia, como se menciona en Vicente de Vera y Gabari Gambarte (2019); según sea su percepción y lo resistente de su personalidad (González-García et al., 2017), dada su situación familiar, profesional o el tipo de contrato.

Luego, entonces, la pregunta es: ¿cómo los aspectos personales, sociales, profesionales y de la labor docente, así como institucionales, conforman la alta resiliencia del profesorado universitario? Por lo que el objetivo de este artículo es dar a conocer cómo el profesorado universitario, de las áreas de estudio investigadas, conforma su alta resiliencia, con la finalidad de que tanto docentes como las IES puedan disminuir los factores de riesgo y promover mecanismos que les permitan mantener altos niveles de resiliencia y una mayor permanencia en la labor docente.

\section{Metodología}

El presente artículo se realizó como parte de un trabajo de tesis doctoral de carácter mixto. Este presenta los hallazgos de la parte de corte cualitativo del estudio de casos con un alcance descriptivo el cual, según Maxwell et al. (2015), es importante cuando se trata de medir fenómenos complejos, como la resiliencia, ya que los datos de carácter subjetivo y cualitativo proveen un entendimiento más detallado de las relaciones dinámicas que explican las variantes en el bienestar después de la exposición a golpes o estresores de vida. Así mismo, Liebenberg y Ungar (2009) señalan que la comprensión de la resiliencia es altamente compleja porque su

6

Teresa de Jesús Sierra-Molina y Dora Esperanza Sevilla-Santo

Los artículos de la Revista Electrónica Educare del Centro de Investigación y Docencia en Educación de la Universidad Nacional, Costa Rica, se comparten bajo términos de la Licencia Creative Commons: Reconocimiento, No Comercial, Sin Obra Derivada 3.0 Costa Rica. Las autorizaciones adicionales a las aquí delimitadas se pueden obtener en el correo: educare@una.cr 
http://doi.org/10.15359/ree.25-3.16

estudio: a) incluye tanto factores genéricos que se presentan en diferentes poblaciones, así como las percepciones de los individuos acerca de los recursos de su entorno y los personales, b) se requiere encontrar formas para documentar con precisión la interacción individuo-ambiente, c) se requiere su comprensión tanto como un producto como un proceso, ya que, d) es, en sí mismo, dinámicamente negociado y construido en el marco de una cultura y contexto específico.

En este trabajo se tomaron en cuenta ocho casos de profesorado universitario, los cuales participaron de manera voluntaria, aunque debían contar con las siguientes características: ser profesorado activo y tener al menos dos años de antigüedad como profesores o profesoras universitarios. Las ocho personas participantes, todas casadas y con hijos, habían sido identificadas en la primera parte del estudio original con una alta resiliencia, obtenida a través de la Escala de resiliencia mexicana (RESI-M de Palomar Lever y Gómez Valdez, 2010), quienes fungieron como docentes en escuelas públicas (cinco) y privadas (tres), cuatro de ingeniería y cuatro de psicología, de una ciudad del sureste de México, durante el ciclo escolar enero-junio 2018. En la Tabla 1 se presentan los datos de los sujetos participantes.

Tabla 1: Profesoras y profesores participantes

\begin{tabular}{ccccccccc}
\hline Caso & Género & $\begin{array}{c}\text { Edad } \\
\text { (años) }\end{array}$ & $\begin{array}{c}\text { Estado } \\
\text { civil }\end{array}$ & $\begin{array}{l}\text { Carrera en } \\
\text { la que es } \\
\text { docente }\end{array}$ & $\begin{array}{c}\text { Años } \\
\text { ejerciendo } \\
\text { la docencia }\end{array}$ & $\begin{array}{l}\text { Tipo de } \\
\text { contratación }\end{array}$ & $\begin{array}{l}\text { Tipo de IES en } \\
\text { la que labora }\end{array}$ & $\begin{array}{c}\text { Nivel de } \\
\text { resiliencia } \\
\text { identificado }\end{array}$ \\
\hline 1 & Mujer & 39 & Casada & Psicología & 15 & $\begin{array}{l}\text { Tiempo } \\
\text { completo }\end{array}$ & Pública & Alta \\
2 & Mujer & 59 & Casada & Psicología & 22 & $\begin{array}{l}\text { Tiempo } \\
\text { completo }\end{array}$ & Privada & Alta \\
3 & Mujer & 48 & Casada & Ingeniería & 8 & $\begin{array}{l}\text { Tiempo } \\
\text { completo }\end{array}$ & Pública & Alta \\
4 & Hombre & 65 & Casado & Psicología & 43 & $\begin{array}{l}\text { Tiempo } \\
\text { completo }\end{array}$ & Pública & Alta \\
5 & Hombre & 62 & Casado & Psicología & 34 & $\begin{array}{l}\text { Tiempo } \\
\text { completo }\end{array}$ & Pública & Media \\
6 & Hombre & 59 & Casado & Ingeniería & 35 & $\begin{array}{l}\text { Por horas } \\
\text { Tiempo }\end{array}$ & Privada & Alta \\
7 & Hombre & 58 & Casado & Ingeniería & 36 & $\begin{array}{l}\text { Píba } \\
\text { completo }\end{array}$ & Alta \\
8 & Hombre & 50 & Casado & Ingeniería & 22 & $\begin{array}{l}\text { Tiempo } \\
\text { completo }\end{array}$ & Privada & Alta \\
\hline
\end{tabular}

Nota: Elaboración propia. 
Del grupo de participantes, un sujeto se había jubilado como docente de tiempo completo recientemente (el único que en ese momento se encontraba laborando como profesor por horas); uno podía haber optado por jubilarse, pero seguía trabajando y otro más estaba a un mes de la jubilación. Dos profesores y una profesora más se encontraban a unos pocos años de llegar a la edad de jubilarse, lo que da cuenta de la larga permanencia en funciones de estas personas. Por último, dos profesoras estaban aún a medio camino de la edad de retirarse, ambas tenían hijos a pequeños de quienes hacerse cargo.

Se utilizó una entrevista a profundidad con cada profesor y profesora, cuyas preguntas se derivaron de categorías identificadas en el acercamiento previo de corte cuantitativo.

Para la parte cualitativa se derivaron ocho preguntas con las que fue posible ahondar, ya que el personal docente contestó compartiendo sus historias, sus experiencias y recuerdos desde sus primeros años como docentes, hasta la descripción de sus condiciones actuales. Las experiencias y comentarios compartidos se codificaron de la siguiente manera: la primera letra $(P)$ significa profesor o profesora, la segunda corresponde al género de cada docente ( $\mathrm{M} \mathrm{u} \mathrm{H}$ : mujer u hombre), la tercera hace referencia a la carrera en la que se desempeña como docente (I o P: Ingeniería o Psicología) y la cuarta y quinta hacen referencia al tipo de IES (PU o PR: pública o privada).

A partir de las narraciones que el personal docente compartió se identificaron, en un primer momento, aspectos que pudieron agruparse en: a) personales, b) sociales, c) profesionales y de la labor docente; así como d) institucionales. Luego se procedió a identificar, desde el significado que cada docente le concedía, si estos aspectos eran percibidos como factores protectores o de riesgo a su labor y permanencia en la docencia, al tiempo de que también fue posible clasificar estos como factores internos o externos, de acuerdo con la teoría.

\section{Resultados y discusión}

Como se ha comentado en la primera parte de este trabajo, ser personal docente universitario hoy en día es mucho más complejo que en épocas pasadas. Si además se tiene en cuenta que el avance de las ciencias y las tecnologías de la información es cada vez más acelerado, es necesario conocer cómo se conforma la alta resiliencia de aquel profesorado que ha logrado permanecer por muchos años en la labor docente y realizar cambios a su labor una y otra vez para incorporar las innovaciones solicitadas por sus instituciones; y analizar si existe alguna combinación o conjunción de aspectos internos (personales y de la labor docente) percibidos por el profesorado, como de apoyo o riesgo (externo) que identifiquen de su entorno familiar, social y del contexto de la misma institución.

A continuación, se presenta la forma en la que se identifican los aspectos que conforman la resiliencia del personal docente. Los tamaños de las flechas en la Figura 1 reflejan, de manera proporcional, la acumulación de las respuestas de las personas participantes. Se presenta, así mismo, la descripción de la forma en la que se perciben los aspectos antes mencionados (como 
http://doi.org/10.15359/ree.25-3.16

http://www.una.ac.cr/educare educare@una.ac.cr

factores protectores o de riesgo, internos o externos) en forma ascendente, comenzando con aquellos que fueron menos percibidos (los aspectos sociales, en flecha café oscuro) a aquellos más mencionados (los profesionales y de la labor docente, en flechas de color blanco), como se puede apreciar en la Figura 1.

Figura 1: La alta resiliencia del profesorado universitario

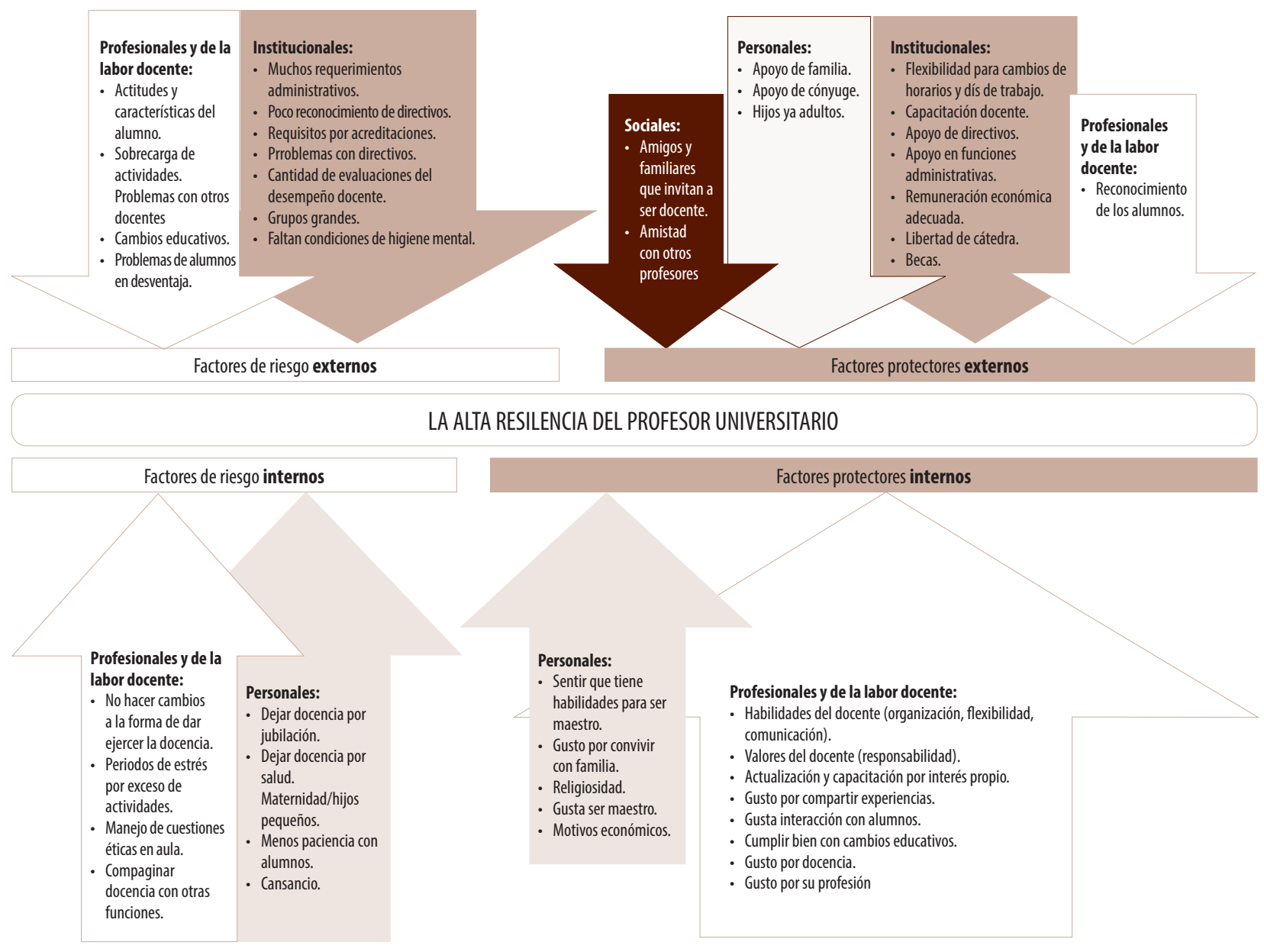

Nota: Elaboración propia.

\section{Aspectos sociales}

Como se puede observar en la Figura 1, la percepción de estos (en flecha café oscuro) funge, en estos casos, solamente como un factor protector externo, ya que se identifica como importante para llegar a la docencia el ser conciente de que se tiene (Grotberg 1996) 
http://doi.org/10.15359/ree.25-3.16

http://www.una.ac.cr/educare

educare@una.ac.cr

amigos, amigas y familiares que invitan a ser docente; sobre todo, en el contexto específico de profesionales que son docentes en ingeniería (Ungar, 2003) como en este caso:

Lo que pasa es que, en la facultad, junto a mi oficina está la oficina de otro ingeniero... él me llamó y me dijo 'oye, necesitan un maestro de matemáticas, ahí está nuestro alumno [... q que fue nuestro alumno de ingeniería, ahí está él y están dando clases también, ¿no te interesa?' ... (PHIPU)

En un par de casos de docentes de tiempo completo, se identifica también como importante el contar con la amistad de otro profesor o profesora; ya que como mencionan Madariaga et al. (2014), estas amistades ayudan a resignificar las vivencias, los cambios y los retos que la docencia actual y las instituciones demandan, ejemplo de esto el siguiente comentario:

... creas una relación de amistad con todos los maestros y en mi caso particular, entre los maestros hay exalumnos míos entonces pues esa relación también es importante...(PHIPR)

\section{Aspectos personales}

Este es el siguiente grupo de aspectos percibidos (en flechas de color beige). Como se puede observar, la mayoría de estos aspectos son identificados como factores protectores; entre los que identifican Wong-Parodi et al. (2015) recursos internos como: la religiosidad, el gusto por la docencia y motivos económicos; así como el contar con una buena autoestima (Melillo, 2004) y sentir que se tiene habilidades para ser maestro o maestra (Grotberg 1996); igualmente el gusto por convivir con familiares, como se refleja en el comentario de una profesora de tiempo completo:

Lo que sí me queda muy claro, es que yo una vez que me instalo acá [en la universidad], cierro la empresa de mi casa y abro la de acá, ¿no? Y cuando estoy en casa estoy dedicada a ellos totalmente. Son pocos los tiempos de lunes a viernes, pero... estoy allá con la familia. No queremos perder esa dinámica de que todavía nos podemos sentar a comer todos, la mayor parte de los días de la semana... (PMIPR)

Entre los aspectos personales que fungen como factores protectores externos se encuentra la percepción de tener apoyo de la familia (Sierra Molina et al., 2019); sobre todo de los hijos e hijas que ya son personas adultas y, como lo mencionaron todos los profesores varones, de las esposas. A continuación, un ejemplo de esto:

... ella [esposa] está consciente y me ha apoyado mucho en ese aspecto y hasta la fecha mis hijos que ya están grandes, cuando tenemos alguna reunión... ¿saben qué? les digo, yo creo que voy a llegar tarde a esta reunión porque tengo que hacer tal actividad, ya sea en el 
http://doi.org/10.15359/ree.25-3.16

diplomado, por las mismas materias, por ambos lados; tanto en la facultad, como en la prepa y ellos son muy conscientes 'no papá, si tiene usted que hacer este reporte, que lo haga, no importa, puede llegar tarde o si no, no importa'... (PHIPU)

La situación personal mencionada por siete de las ocho personas participantes, quienes se desempeñan como docentes de tiempo completo, es la importancia concedida al apoyo del cónyuge para poder realizar todo lo que la labor docente actual les requiere. Ejemplo de lo anterior es el siguiente comentario que, aunque expresado con sentido del humor, refleja la realidad percibida y resignificada (Simpson, 2008) por uno de los participantes:

... lo que tengo que cuidar es que mi esposa no le vaya a cambiar la chapa a la puerta [porque llega muy tarde]... Pobrecita, es que ella... lo normal, me quedo aquí [en la universidad] un poquito más... y a veces cuando hay que hacer investigación, salir al escenario, al campo. Si es usted creyente, rece por mi familia y con buenos deseos... (PHPPU)

Otro participante comenta a este mismo respecto:

Ese es un apoyo muy fuerte de parte de mi esposa que me permite continuar tanto con el trabajo, como continuar con la docencia. Los lunes y los miércoles, por ejemplo, son días que no voy a comer a casa. Ella sabe que el lunes no voy porque tengo clase en la [universidad], entonces aprovecho la hora de la comida para afinar algunos detallitos de la clase... (PHIPR)

Por otro lado, se presenta también la situación de que algunos aspectos personales son percibidos como factores de riesgo interno, entre los cuales se identifica el estar ya cerca de la edad de jubilación, la posibilidad de abandonar la docencia por cuestiones de salud. Esto por la que la resistencia personal pudiera estar disminuida por la edad (González-García et al, 2017). En las profesoras participantes, la maternidad es lo que todas identifican como un riesgo a su labor; sobre todo cuando los hijos o hijas son aún infantes. Situación que también fue mencionada por tres profesores varones, quienes reconocen que cuando los hijos e hijas eran pequeños o pequeñas, era más complejo compaginar la docencia con la convivencia con la familia.

Yo por convicción, también personal, pues yo trataba de estar muy cerca de ellas [de sus hijas], yo las iba a llevar a la escuela, las iba a buscar y varias de estas cosas demandaban también tiempo y pues sí el cómo combinar para poder estar cumpliendo mis tareas docentes fue tal vez diría yo la etapa más difícil, pero no en sí por la docencia sino como una consecuencia o algo relacionado con la docencia... (PHPPU)

Se identifica también el cansancio acumulado por los años de labor y la edad, lo que trae consigo menos paciencia con el alumnado y coincide con la baja de efectividad y compromiso que se puede presentar, según Brooks (2016). Situaciones que, en este grupo de profesoras y 
http://doi.org/10.15359/ree.25-3.16

http://www.una.ac.cr/educare

educare@una.ac.cr

profesores, además refuerzan lo identificado por Hong (2012, en Olivares-Faundéz y Villalta, 2015), cuando mencionan que la resiliencia en muchos casos explica la permanencia en la docencia, podemos decir ahora, a pesar de los varios riesgos mencionados.

\section{Aspectos institucionales}

El tercer grupo de aspectos identificados, son los aspectos institucionales (en flechas café claro), los cuales como se puede observar, fungen como factores protectores y de riesgo en casi igual medida, siendo la percepción de estos como riesgo, ligeramente mayor.

Los aspectos que fungen como factores de riesgo externos son percibidos en forma de muchos requerimientos administrativos como se puede ver en este comentario.

... el día a día de la institución es difícil, en la parte administrativa cada vez hay más exigencia, cada vez está uno más expuesto a reclamos de los alumnos por cosas que realmente no son responsabilidad de uno y pues esa exigencia, ¿no? Y a veces un poquito de rigidez en el sistema administrativo... (PHIPR)

Se perciben también como riesgos institucionales externos el poco reconocimiento por parte de personas directivas, los muchos requisitos que se derivan de los procesos de acreditación, los problemas con personas directivas, la cantidad de evaluaciones que se hace a su desempeño docente (como consecuencia de los procesos de calidad), que como identifican Price et al. (2012) y Vicente de Vera y Gabari Gambarte (2019) les puede desanimar y ponen en evidencia las teorías implícitas y explícitas que tiene el personal docente con respecto a los procesos de acreditación y las evaluaciones (Monereo y Domínguez, 2014). Esto se ve reflejado en comentarios de dos profesoras de tiempo completo de IES públicas:

... hay mucho trabajo extra clase, mucha preparación y a nosotros por ejemplo nos piden, no sé si en otras universidades, pero nosotros necesitamos tener un desempeño promedio cada año... (PMIPU)

... la tendencia de la universidad pública es cada vez como que exigir más y más estándares y más criterios... de que comencé a trabajar a ahora... ha sido una cuestión, así como que... tremenda en relación a la carga administrativa que se tiene que hacer y de investigación y cada vez con los rankings y las producciones... entonces, eso es muy agotador muy desgastante... (PMPPU)

Más aún, dos profesores de tiempo completo de una IES pública mencionan que se hacen varias evaluaciones al año de su desempeño como las del Programa para el Desarrollo Profesional Docente (PRODEP) y el Sistema Institucional de Informes y Gestión de los Académicos (SIGA): 
http://doi.org/10.15359/ree.25-3.16

... tenemos que demostrar qué estuvimos haciendo dentro de los cuatro rubros: tutoría, gestión, investigación y docencia; entonces ahí tenemos que decir: tuvimos tantos cursos de capacitación, etc., tantas clases, tantas horas frente a grupo... entonces tenemos que hacer las otras actividades que van ligadas dentro de la docencia... (PMIPU)

Otro profesor de tiempo completo de IES pública señala:

... las instituciones de educación superior, nuestra misma universidad, pues cada vez demandan más de los profesores, actividades para ir subiendo por los reconocimientos, pues que hay que estudiar un posgrado, que hay que estar en el Sistema Nacional de Investigadores (SNI), que hay que publicar y producir y todo esto y además dar las clases; entonces, además las responsabilidades que se pueden tener en la vida personal y todo eso. Entonces, si uno no está alerta ante esto, pues si se pueden generar ciertas situaciones de estrés, de tensión... (PHPPU)

Así mismo, se identifican como factores de riesgo institucionales (externos) los grupos numerosos de estudiantes y, para dos docentes de tiempo completo de instituciones privadas: ... una serie de factores de higiene mental que se necesitan... (PHIPR), lo que se experimenta cuando se comparten espacios reducidos de trabajo con varias personas, por ocho horas diarias o más. Esto último se contrapone a lo identificado por Olivares-Faundéz y Villalta (2015), quienes destacan que los síntomas de burnout se presentan más en el personal docente por horas.

Por otro lado, se percibe también un gran número de situaciones institucionales que se identifican como factores que mitigan los riesgos (Henderson y Milstein, 2003), factores protectores institucionales (externos) de la resiliencia de las personas participantes y, por ende, de su permanencia en la docencia, entre estos: la flexibilidad de la institución y personal directivo (Villalobos Vergara y Assaél Budnik, 2018) para realizar cambios de horarios y la posibilidad de reponer horas de trabajo en momentos distintos (Arnup y Bowles, 2016); situación que es identificada por cinco de las ocho personas participantes; ya que refleja muy bien la relación dinámica (Hendereson Grotberg, 2006) entre este tipo de aspectos y los personales, como se puede ver en el siguiente comentario de una profesora, altamente resiliente:

... me ayuda mucho la administración de la facultad, o sea, la comprensión del personal que está en dirección, que está en secretaría administrativa. O sea, por ejemplo, tengo una situación familiar y no puedo llegar o tengo que llegar tarde u ocurre un viaje familiar, entonces que haya todas estas consideraciones para hacer ajustes... Eso es lo que para mí es el regalo maravilloso que tiene la facultad de psicología yeso me permite a mí hacer mi trabajo y hacerlo con gusto... porque sí sé que de repente hay momentos que administrativamente es agotador por miles de evaluaciones por todos lados, entonces estás sentada frente al escritorio y de la computadora llenando formatos o lo que sea, pero cuando después dices: 'oye, se me presenta esta situación... hay claro que sí, adelante, que no sé qué, te has estado matando por un montón de días... para mí los dos elementos centrales es la institución y los apoyos que te da la institución en donde se pueda ver la reciprocidad... (PMPPU) 
http://doi.org/10.15359/ree.25-3.16

http://www.una.ac.cr/educare

educare@una.ac.cr

De igual manera, se identifica como factor protector institucional (externo) el contar con capacitación docente (Villalobos Vergara y Assaél Budnik, 2018), lo que coincide con lo señalado por Carlos Guzmán (2011) en cuanto al apoyo de personal directivo, apoyo en funciones administrativas, la remuneración económica (señalada por docentes de tiempo completo de instituciones públicas), la libertad de cátedra y el poder tener acceso a becas para continuar con su formación profesional docente (también identificado por docentes de IES públicas).

\section{Aspectos profesionales y de la labor docente}

Por último, en flechas blancas se presentan los aspectos más identificados como significativos para el profesorado en la conformación de su alta resiliencia: los aspectos profesionales y de la labor docente, los cuales, como se puede observar en la Figura 1, al ubicarse en las cuatro esquinas, proveen un balance dinámico ya que son construidos junto con otras personas de su entorno personal e institucional (Liebenberg y Ungar, 2009).

En estos casos, dichos aspectos son, en su mayoría, percibidos como factores protectores internos: el sentir que se cuenta con habilidades para ser docente (las que fueron mencionadas por todas las personas participantes), el contar con valores como la responsabilidad y el compromiso por mantenerse actualizados los conocimientos por interés propio, el gusto por compartir sus experiencias con otras personas y la interacción con el alumnado:

... ¿Por qué me gusta?, número uno. Una vez me preguntaron que si ya no construía, que si ya no me dedico a la construcción como ingeniera civil y les dije que sí, solo que ahora construía ingenieros... En verdad, la labor de la docencia, se me figura que es o siento que es algo muy bonito, no solo es enseñarles tu materia, tu asignatura, sino que en forma conjunta los estamos preparando para la profesión, entonces, me gusta mucho y creo que es una gran responsabilidad y gran labor, entonces hay que estar al 100 con los muchachos y además de que siempre estas estudiando y aprendiendo... (PMIPU)

Se identifican también como factores protectores internos profesionales y de la labor docente, el compromiso por cumplir adecuadamente con los cambios educativos, el gusto por la docencia y por su profesión. Todos estos aspectos que coinciden con las tres fuentes de la resiliencia descritas porGrotberg (1996) y los pilares descritos por Melillo (2004), respectivamente: sentir que se es un profesor o profesora responsable (con una moral e iniciativa), que se tiene la disposición para trabajar con el alumnado (capacidad para relacionarse), que se puede trabajar con el alumnado y responder a los retos de la profesión docente (autoestima, creatividad y pensamiento crítico).

Le siguen en cantidad, los percibidos como factores de riesgo externo como las actitudes y características del alumnado de hoy en día, la sobrecarga de actividades percibida, los problemas con otros profesores y profesoras, los cambios educativos (entendidos como consecuencias de las reformas e innovaciones), ejemplo de esto: 
http://doi.org/10.15359/ree.25-3.16

Además de que en 6 años ya hemos tenido dos cambios de programa... además... y ahí si nos vimosobligados.... Nosotros trabajamos sobre un proyecto práctico y ese proyecto, es a materia ha ido evolucionando, como por ejemplo la materia de licenciatura que es Procedimientos de construcción, ha ido evolucionando, porque a través del tiempo hemos ido identificando lagunas de conocimiento. Entonces cuando llegan ha habido que ir adaptando para que los muchachos salgan con más y más habilidades... (PMIPU)

También, el tener que hacer frente a casos de alumnado con problemas o en situaciones de desventaja, lo que es percibido como altamente retador (Mérida Serrano, 2006) y hasta como lo más difícil que algunos o algunas han enfrentado como docentes, dado que no siempre se tiene formación como tutores y tutoras o para afrontar situaciones de índole emocional, como se puede ver en la siguiente experiencia:

... de las que te puedo decir es cuando detecto que algún alumno ha faltado a clase y luego le sigo la huella, por algún motivo pregunto, indago en Control Escolar, ¿qué pasó? Y pues está en una situación de desventaja bastante fuerte, o sea problemas familiares, problemas económicos, problemas jurídicos de repente que no pueden resolver, entonces si me ha tocado ser parte como en un chico que estuvo involucrado en un asesinato... o sea, cosas muy fuertes, que afortunadamente salió avante, estuvo en arraigo y demás... Enfermedades de chavos... que muchas veces estás atado de manos y no le puedes ayudar pero para nada... pero si puedes sentir empatía con ellos y saber que pueden acudir a ti para ponerse al día o para algún consejo... yo creo que esas han sido las situaciones más difíciles... (PMIPU)

Le siguen luego aspectos profesionales y de la labor docente que son percibidos como factores de riesgo interno, entre los cuales el más mencionado es el no llevar a cabo los cambios requeridos a la propia práctica docente, lo que pone de manifiesto a un tipo de docente (Hargreaves 1994), ejemplo de esto los comentarios de dos profesores:

... sigo dando clases de la misma forma que mis profesores me dieron clase a mí... no uso prácticamente ni un tipo de recursos audiovisuales, por ejemplo, no uso rotafolios, no uso computadora... bueno, sí uso computadora, pero no es parte de... (PHIPR)

O sea, cumplo con los requerimientos institucionales así, literalmente, o sea, me pides esto... te lo doy, ajá, pero que me guste... no me gusta y que lo hago bien... si lo hago bien (PMPPU)

Le sigue en importancia el identificar que existen períodos de estrés muy fuertes, como los finales de semestre en los que se conjugan muchas actividades tanto con el alumnado, propias del cierre de las asignaturas, como de sus otras funciones dentro de la institución; esto pone de manifiesto sus teorías implícitas acerca de su labor y la institución (Monereo y Domínguez, 2014). Luego, se mencionó el manejo de cuestiones éticas con el alumnado, las cuales, aunque se identifican como muy importantes, se reconoce que consumen mucho tiempo. 
Por último, el aspecto profesional y de la labor docente que es percibido como factor protector externo por cinco del grupo de ocho docentes es el contar con el reconocimiento del alumnado, situación que, según su criterio, equilibra o contrarresta los factores de riesgo antes mencionados; manifiestan que es por este reconocimiento que vale la pena ser profesor o profesora y seguir en la docencia, como da cuenta la siguiente respuesta:

Participar en la formación de los alumnos y porque te das cuenta que son seres humanos que necesitan esa dirección, no solo de la materia que les impartes, sino es muy gratificante cuando un alumno se acerca a ti, después de que pasaste por su fase de preparación y alguna cosa que les dijiste que a veces ni tenía que ver con la materia, algún consejo, algún apoyo, algún algo, pues fue punto clave de motivación para ellos. Me he topado con casos de unos que se acercan a decírtelo. Entonces, pues con eso estoy más que pagada... o sea, ya, esa es la verdadera retribución de un profesor... (PMIPU)

\section{Conclusiones}

Como se ha podido constatar, desde las experiencias e historias personales y profesionales, los aspectos más importantes en la conformación de la alta resiliencia del personal docente son, en estos casos, en número y dinamismo, los aspectos profesionales y de la labor docente ya que, como se ha podido apreciar, se encuentran generando un balance dinámico como factores protectores y de riesgo tanto internos como externos. Además, se observa una bidireccionalidad de estos mismos, dado que, junto con los aspectos personales, se perciben como factores protectores internos que contrarrestan los efectos de los aspectos profesionales y de la labor decente, más los institucionales que son identificados como factores de riesgo externos.

Con respecto a los aspectos institucionales, se puede también constatar la dinámica bidireccional, ya que son percibidos tanto como factores de riesgo como factores protectores que mitigan y facilitan la permanencia en la docencia aún y cuando hay cada vez más presiones y requisitos con los que cumplir. Ello corrobora lo encontrado por otras investigaciones en cuanto a la importancia de que las instituciones puedan promover mecanismos encaminados a disminuir los factores de riesgo y el burnout, y facilitar aquellos que permitan mantener altos niveles de resiliencia, lo que se puede ver reflejado en una mayor permanencia en la labor docente.

En tercer lugar de importancia se encuentran los aspectos personales, ya que en su mayoría funcionan como factores protectores tanto internos como externos, los que a su vez protegen de los personales que son percibidos como factores de riesgo internos, como el ser madre de hijas e hijos pequeños y compaginar con la docencia de tiempo completo, realidad de una gran cantidad de docentes en educación superior actualmente.

Los aspectos sociales son, por su parte, solo percibidos como factores protectores externos por docentes del área de ingeniería, ya que es gracias a amistades que les invitan y animan, que llegan a la labor docente; esto, dado a que su formación resulta alejada de la requerida para 
http://doi.org/10.15359/ree.25-3.16

ejercer la docencia. Sería pertinente indagar, si estos aspectos tienen igual significancia en la conformación de la resiliencia y permanencia de docentes de otros campos del conocimiento.

Entonces, ¿cómo se conforma la alta resiliencia del profesorado universitario? Desde las voces de docentes participantes se osberva una conformación dinámica en la que aspectos personales, sociales, profesionales y de la labor docente; así como institucionales, son percibidos tanto como factores protectores como de riesgo internos y externos. Esto corrobora que al profesorado universitario participante se le puede ver como a personas en alta resiliencia (Quintero Velásquez, 2005). Desde la perspectiva triádica de la resiliencia (Capanna et al., 2015), esto puede explicar su permanencia en la docencia hasta la edad de jubilación y más allá. Para esto se requiere percibir en el ambiente familiar, el institucional (Ungar 2003) y en Isa propia persona, mayor cantidad de factores protectores que mitiguen los muchos aspectos identificados como factores de riesgo, sobre todo externos.

Los resultados, entonces, corroboran también la importancia de contar con pilares de la resiliencia (Melillo, 2004) como autoestima, creatividad, moralidad y pensamiento crítico, y de ser capaces de identificar las fuentes de la resiliencia (Grotberg, 1996) como reconocer que se es un profesor o profesora capaz (con numerosos recursos y aspectos profesionales y de la labor docente internos), que se tiene el apoyo de familiares y la institución en momentos de sobrecarga y cuando hay situaciones personales y familiares con las que compaginar la docencia (Wong-Parodi et at, 2015); pero que también se puede hacer frente a los muchos retos que la educación superior de hoy en día implican para la persona profesional que labora como profesor y profesora.

\section{Declaración de Material complementario}

Este artículo tiene disponible, como material complementario:

-La versión preprint del artículo en https://doi.org/10.5281/zenodo.4796140

\section{Referencias}

Arnup, J. y Bowles, T. (2016). Should I stay or should I go? Resilience as a protective factor for teachers' intention to leave the teaching profession. Australian Journal of Education, 60(3), 229-244. https://doi.org/10.1177\%2F0004944116667620

Beigi, S. (2016). The timeline of resilience: A roadmap for cross operationalization of resilience. https://www.academia.edu/21541954/THE_TIMELINE_OF_RESILIENCE_A ROADMAP FOR CROSS OPERATIONALIZATION OF RESILIENCE

Benítez-Corona, L. y Barrón-Tirado, M. C. (2018). Análisis cualitativo de la resiliencia en estudiantes de postgrado. Revista electrónica Educare, 21(1), 1-21. https://doi.org/10.15359/ree.22-1.7 
http://doi.org/10.15359/ree.25-3.16

http://www.una.ac.cr/educare

educare@una.ac.cr

Brooks, C. (2016). Teacher subject identity in professional practice. Teaching with a professional compass. Routledge. https://doi.org/10.4324/9781315774770

Capanna, C., Stratta, P., Hjemdal, O., Collazoni, A. y Rossi, A. (2015). The Italian validation study of the Resilience Scale for Adults (RSA). Aplied Psychology Bulletin, 63(272), 16-24. https:// www.academia.edu/18398592/The_Italian_validation_study_of the_Resilience_Scale_ for Adults RSA

Carlos Guzmán, J. J. (2011). ¿Cómo se forman los profesores efectivos de educación superior? Análisis de las fases de su desarrollo docente. XI Congreso Nacional de Investigación Educativa. Consejo Mexicano de Investigación Educativa, México. http://www.comie.org. $\mathrm{mx} /$ congreso/memoriaelectronica/v11/docs/area 15/1985.pdf

González-García, H., Pelegrín, A. y Carballo, J. (2017). Ira y personalidad resistente en deportistas de raqueta y resistencia. Revista Iberoamericana de Ciencias de la Actividad Física y el Deporte, 6(2), 21-29. https://doi.org/10.24310/riccafd.2017.v6i2.3776

Grotberg, E. H. (1996). The international resilience project findings from the research and the effectiveness of interventions. Paper presented at the Annual Convention of the International Council of Psychologists. Banff, Canada. http://files.eric.ed.gov./fulltext/ED419584.pdf

Hargreaves, A. (1994). Dissonant voices: Teachers and the multiple realities of restructuring. Conferencia presentada en Annual Meeting of the American Educational Research Association, New Orleans. http://files.eric.ed.gov/fulltext/ED372056.pdf

Henderson, N. y Milstein, M. M. (2003). Resiliencia en la escuela. Paidós.

Henderson Grotberg, E. (2006). La resiliencia en el mundo de hoy. Cómo superar las adversidades. Gedisa.

Liebenberg, L. y Ungar, M. (2009). 1 Introduction: The challenges in researching resilience. En L. Liebenberg y M. Ungar (Eds.), Researching resilience (pp. 3-25). University of Toronto Press. https://doi.org/10.3138/9781442697669

Madariaga, J. M., Palma, M. de las O., Surjo, P., Villalba, C. y A. Arribillaga, A. (2014). 1 La construcción social de la resiliencia. En J. Madariaga (Coord.), Nuevas miradas sobre la resiliencia. Ampliando ámbitos y prácticas (pp. 11-30). Gedisa.

Maxwell, D., Constas, M., Frakenberger, T., Klaus, D. y Mock, M. (2015). Qualitative data and subjective indicators for resilience measurement. Resilience Measurement Technical Working Group (Technical Series No. 4). Food Security Information Network. https://www. fsinplatform.org/sites/default/files/paragraphs/documents/FSIN_TechnicalSeries_4.pdf

Melillo, A. (2004). Sobre la necesidad de especificar un nuevo pilar de la resiliencia. En A. Melillo, E. N. Suárez Ojeda y D. Rodríguez (Comps.), Resiliencia y subjetividad. Los ciclos de la vida (pp. 77-90). Paidós. 
http://doi.org/10.15359/ree.25-3.16

http://www.una.ac.cr/educare educare@una.ac.cr

Mérida Serrano, R. (2006). Nueva percepción de la identidad profesional del docente universitario ante la convergencia europea. Revista Electrónica de Investigación Educativa, 8(1), 1-18. http://www.redalyc.org/articulo.oa?id=15508105

Monereo, C. y Domínguez, C. (2014). La identidad docente de los profesores universitarios competentes. Educación XX1, 17(2), 83-104. https://doi.org/10.5944/educxx1.17.2.11480

Olivares-Faúndez, V. y Villalta, P. (2015). Burnout y resiliencia en profesores universitarios. En L. Godoy y E. Ansoleaga (Comps.), Un campo en tensión o tensión entre campos. Psicología de las organizaciones y del trabajo en Iberoamérica (pp. 307-319). RIL Editores.

Palomar Lever, J. y Gómez Valdez, N. E. (2010). Desarrollo de una escala de medición de la resiliencia con mexicanos (RESI-M). Interdisciplinaria, 27(1), 7-22. https://www.redalyc.org/ pdf/180/18014748002.pdf

Price, A., Mansfield, C. y McConney, A. (2012). Considering 'teacher resilience' from critical discourse and labour process theory perspectives. British Journal of Sociology of Education, 33(1), 81-95. https://doi.org/10.1080/01425692.2011.614748

Quintero Velásquez, A. M. (2005). Resiliencia: Contexto no clínico para trabajo social. Revista Latinoamericana de Ciencias Sociales, Niñez y Juventud, 3(1), 73-94. http://www.scielo.org. co/scielo.php?script=sci arttext\&pid=S1692-715X2005000100004\&lng=en\&tlng=es

Sierra Molina, T. de J., Sevilla Santo, D. E. y Martín Pavón, M. J. (2019). Profesor universitario, ser en resiliencia: Una mirada a su labor en el contexto educativo actual. Diálogos sobre educación, 10(19). http://dialogossobreeducacion.cucsh.udg.mx/index.php/DSE/article/ view/539

Simpson, M. G. (2008). Resiliencia en el aula, un camino posible. Bonum.

Ungar, M. (2003). Qualitative contributions to resilience research. Qualitative Social Work, 2(1), 85-102. https://doi.org/10.1177/1473325003002001123

Vicente de Vera, M. I. y Gabari Gambarte, M. I. (2019). Burnout y factores de resiliencia en docentes de educación secundaria. International Journal of Sociology of Education, 8(2), 127-152. http://dx.doi.org/10.17583/rise.2019.3987

Villalobos Pérez-Cortés, M. (2009). Resiliencia: Cómo aceptar y adaptarse a los cambios. Una propuesta educativa. Minos Tercer Milenio.

Villalobos Vergara, P. y Assaél Budnik, J. (2018). Dimensiones individuales, relacionales e institucionales en la resiliencia de profesores principiantes egresados de una universidad pública. Psicoperspectivas. Individuo y Sociedad, 17(1), 1-11. https://doi.org/10.5027/ psicoperspectivas-vol17-issue1-fulltext-1145

Wong-Parodi, G., Fischhoff, B. y Strauss, B. (2015). Resilience vs. adaptation: Framing and action. Climate Risk Management, 10, 1-7. https://doi.org/10.1016/j.crm.2015.07.002 